\title{
A NOTE ON RATIONAL NORMAL SCROLLS
}

\author{
MARGHERITA BARILE
}

\begin{abstract}
We give a general upper bound for the arithmetical rank of the ideals generated by the 2-minors of scroll matrices with entries in an arbitrary commutative unit ring.
\end{abstract}

Introduction. Given a field $K$, consider the integer $d \geq 2$ and the positive integers $n_{1}, \ldots, n_{d}$. Set $N=d-1+\sum_{i=1}^{d} n_{i}$. The projective variety $S_{n_{1}, \ldots, n_{d}}$ of $\mathbf{P}_{K}^{N}$ defined by the vanishing of all 2-minors of the matrix of indeterminates

$A=\left(\begin{array}{llll|l|llll}X_{1,0} & X_{1,1} & \cdots & X_{1, n_{1}-1} & \cdots & X_{d, 0} & X_{d, 1} & \cdots & X_{d, n_{d}-1} \\ X_{1,1} & X_{1,2} & \cdots & X_{1, n_{1}} & \cdots & X_{d, 1} & X_{d, 2} & \cdots & X_{d, n_{d}}\end{array}\right)$

is called a rational normal scroll. It is irreducible, and its dimension is equal to $d$. In [1], Badescu and Valla show that the arithmetical rank of each of these varieties, i.e., the least number of homogeneous equations needed to define this variety set-theoretically, is equal to $N-2$. In their paper, they explicitly give $N-2$ defining equations $F_{i}=0$, $i=1, \ldots, N-2$, where $F_{1}, \ldots, F_{N-2}$ are homogeneous polynomials of $K\left[X_{1,0}, \ldots, X_{1, n_{1}}, \ldots, X_{d, 0}, \ldots, X_{d, n_{d}}\right]$, and they show that the set of points of $\mathbf{P}_{K}^{N}$ where all $F_{1}, \ldots, F_{N}$ vanish is $S_{n_{1}, \ldots, n_{d}}$. If $K$ is an algebraically closed field, from Hilbert's Nullstellensatz, we know that this statement is equivalent to the equality between the following two ideals of $K\left[X_{1,0}, \ldots, X_{1, n_{1}}, \ldots, X_{d, 0}, \ldots, X_{d, n_{d}}\right]$ : one is the ideal generated by all 2-minors of $A$, (which coincides with the defining ideal of $S_{n_{1}, \ldots, n_{d}}$, i.e., the ideal generated by all homogeneous polynomials vanishing at all its points), the other is the radical of the ideal generated by $F_{1}, \ldots, F_{N-2}$.

In the present paper, we give a ring-theoretical generalization of this result. We show that the two ideals still coincide when the algebraically

2010 AMS Mathematics subject classification. Primary 13A15, 14J26, 14 M10.

Keywords and phrases. Arithmetical rank, rational normal scrolls.

Received by the editors on August 26, 2014, and in revised form on November 25, 2014. 
closed field $K$ is replaced by any commutative unit ring $R$. This, which, of course, remains true if the indeterminates are replaced by arbitrary elements of $R$, means that the arithmetical rank of the first ideal is always at most $N-2$. In this way, we also obtain an alternative proof of $[\mathbf{1}$, Theorem 4.1].

1. Preliminary results. Let $R$ be a commutative unit ring. Given $d$ positive integers $n_{1}, \ldots, n_{d}$, let $D=D_{n_{1}, \ldots, n_{d}}$ be the ideal of $R$ generated by the 2-minors of the following matrix of indeterminates over $R$ :

$$
A=\left(\begin{array}{llll|l|llll}
X_{1,0} & X_{1,1} & \cdots & X_{1, n_{1}-1} & \cdots & X_{d, 0} & X_{d, 1} & \cdots & X_{d, n_{d}-1} \\
X_{1,1} & X_{1,2} & \cdots & X_{1, n_{1}} & \cdots & X_{d, 1} & X_{d, 2} & \cdots & X_{d, n_{d}}
\end{array}\right) .
$$

For all indices $i=1, \ldots, d$, every 2 -minor of the submatrix

$$
\left(\begin{array}{llll}
X_{i, 0} & X_{i, 1} & \cdots & X_{i, n_{i}-1} \\
X_{i, 1} & X_{i, 2} & \cdots & X_{i, n_{i}}
\end{array}\right)
$$

will be called an $(i)$-minor. The set of $(i)$-minors is empty whenever $n_{i}=1$. All these minors will be called pure. For all indices $i, j$ such that $1 \leq i<j \leq d$, every non-pure 2 -minor of the submatrix

$$
\left(\begin{array}{llll|llll}
X_{i, 0} & X_{i, 1} & \cdots & X_{i, n_{i}-1} & X_{j, 0} & X_{j, 1} & \cdots & X_{j, n_{j}-1} \\
X_{i, 1} & X_{i, 2} & \cdots & X_{i, n_{1}} & X_{j, 1} & X_{j, 2} & \cdots & X_{j, n_{j}}
\end{array}\right)
$$

will be called an $(i, j)$-minor.

It is well known, see [2], that, for every index $i$, the radical of the ideal $I_{i}$ of $S$ generated by the set of all $(i)$-minors is equal to the radical of an ideal of $S$ generated by $n_{i}-1$ elements $F_{i, 1}, \ldots, F_{i, n_{i}-1}$ (if $n_{i}=1$, we set $I_{i}=(0)$ ). For all indices $i, j$ such that $1 \leq i<j \leq d$, let $B_{n_{i}, n_{j}}$ be the bridge introduced in [1, page 1648]. We recall its definition. Set $m_{i, j}=\operatorname{lcm}\left(n_{i}, n_{j}\right)$, and let $p_{i, j}$ and $q_{i, j}$ be integers such that $m_{i, j}=p_{i, j} n_{i}=q_{i, j} n_{j}$. For all integers $\alpha$ such that $0 \leq \alpha \leq m_{i, j}$, let $c, r, e$ and $f$ be integers such that $\alpha=c p_{i, j}+r=e q_{i, j}+f$, with $0 \leq r<p_{i, j}, 0 \leq f<q_{i, j}$. Finally, set

$$
\begin{aligned}
B_{n_{i}, n_{j}}\left(X_{i, 0}, \ldots,\right. & \left.X_{i, n_{i}}, X_{j, 0}, \ldots, X_{j, n_{j}}\right) \\
= & \sum_{\alpha=0}^{m_{i, j}}(-1)^{\alpha}\left(\begin{array}{c}
m_{i, j} \\
\alpha
\end{array}\right) X_{i, n_{i}-c}^{p_{i, j}-r} X_{i, n_{i}-c-1}^{r} X_{j, e}^{q_{i, j}-f} X_{j, e+1}^{f} .
\end{aligned}
$$


When using this notation, which is taken from [1], we will always assume that $i<j$. For any monomial $\pi=X_{i, k_{1}}^{\ell_{1}} \cdots X_{i, k_{s}}^{\ell_{s}}$ in the entries of the $i$ th block of $A$ we will call $w(\pi)=\sum_{i=1}^{s} k_{i} \ell_{i}$ the weight of $\pi$. Given an integer $s>0$, and indices $i_{1}<i_{2}<\cdots<i_{s}$, if $\pi_{i_{h}}$ is a monomial in the entries of the $i_{h}$ th block of $A$, and $\pi=\pi_{i_{1}} \pi_{i_{2}} \cdots \pi_{i_{s}}$, then $w(\pi)=\sum_{h=1}^{s} w\left(\pi_{i_{h}}\right)$ is called the weight of $\pi$. Moreover, $\operatorname{deg}_{i_{h}}(\pi)=\operatorname{deg}\left(\pi_{i_{h}}\right)$ will be called the $i_{h^{-}}$degree of $\pi$.

Lemma 1.1. Let $i_{1}$ and $i_{2}$ be integers such that $1 \leq i_{1}<i_{2} \leq d$. Two monomials of $S$ in the entries of the blocks with indices $i_{1}, i_{2}$ are congruent modulo the ideal generated by the 2-minors of the submatrix formed by these blocks if and only if they have the same weight and the same $i_{k}$-degree for $k=1,2$.

Proof. Every $(i)$-minor of $A$ is the difference of two quadratic monomials of $i$-degree 2 , and every $(i, j)$-minor of $A$ is the difference of two quadratic monomials of $i$-degree 1 and $j$-degree 1 . In view of this, the only if part of the claim is easy.

We prove the if part. In order to simplify our notation, we denote the indeterminates of the $i_{1}$ th block by $X_{0}, \ldots, X_{n_{i_{1}}}$ and those of the $i_{2}$ th block by $Y_{0}, \ldots, Y_{n_{i_{2}}}$. We call $I$ the ideal generated by the 2 minors of the submatrix of $A$ formed by these two blocks. Let $\mu$ and $\mu^{\prime}$ be monomials in the first set of variables, $\nu$ and $\nu^{\prime}$ monomials in the second set of variables. Let $w_{1}, w_{1}^{\prime}, w_{2}$ and $w_{2}^{\prime}$ be the weights of $\mu, \mu^{\prime}$, $\nu$ and $\nu^{\prime}$, respectively. Suppose that $\lambda=\mu \nu$ and $\lambda^{\prime}=\mu^{\prime} \nu^{\prime}$ have the same weight $w=w_{1}+w_{2}=w_{1}^{\prime}+w_{2}^{\prime}$ and that $\operatorname{deg}(\mu)=\operatorname{deg}\left(\mu^{\prime}\right)$ and $\operatorname{deg}(\nu)=\operatorname{deg}\left(\nu^{\prime}\right)$. We prove that the monomials $\lambda$ and $\lambda^{\prime}$ are congruent modulo $I$. We proceed by induction on $w$. If $w=0$, then $\lambda$ and $\lambda^{\prime}$ are the same monomial of the form $X_{0}^{i} Y_{0}^{j}$. So assume that $w>0$, and suppose the claim true for all monomials fulfilling the same assumption, but with smaller $w$. Then, up to exchanging the blocks, we have one of the following cases: either $w_{1}>0$ and $w_{1}^{\prime}>0$, or $w_{1}>0$ and $w_{2}^{\prime}>0$. In the first case, $\mu$ and $\mu^{\prime}$ are not pure powers of $X_{0}$; hence, $X_{h}$ divides $\mu$ and $X_{h^{\prime}}$ divides $\mu^{\prime}$ for some $h, h^{\prime} \geq 1$. If $h=h^{\prime}$, then induction applies to $\lambda / X_{h}$ and to $\lambda^{\prime} / X_{h}$, which are thus congruent modulo $I$. Hence, the same holds for $\lambda$ and $\lambda^{\prime}$.

Now assume that $h \neq h^{\prime}$. Let $\bar{\lambda}=X_{h-1} \lambda / X_{h}$ and $\bar{\lambda}^{\prime}=X_{h^{\prime}-1} \lambda / X_{h^{\prime}}$. Then $w(\bar{\lambda})=w\left(\bar{\lambda}^{\prime}\right)=w-1$. Hence induction applies to the monomials 
$\bar{\lambda}$ and $\bar{\lambda}^{\prime}$ so that these are congruent modulo $I$. We thus have

$$
X_{h-1} \lambda / X_{h} \equiv X_{h^{\prime}-1} \lambda^{\prime} / X_{h^{\prime}}(\bmod I),
$$

which implies that

$$
X_{h^{\prime}} X_{h-1} \lambda \equiv X_{h^{\prime}-1} X_{h} \lambda^{\prime} \quad(\bmod I) .
$$

On the other hand, since $X_{h^{\prime}} X_{h-1} \equiv X_{h^{\prime}-1} X_{h}(\bmod I)$, we also have

$$
X_{h^{\prime}} X_{h-1} \lambda \equiv X_{h^{\prime}-1} X_{h} \lambda \quad(\bmod I),
$$

so that, finally

$$
X_{h^{\prime}-1} X_{h} \lambda \equiv X_{h^{\prime}-1} X_{h} \lambda^{\prime} \quad(\bmod I) .
$$

Since $I$ is a prime ideal generated in degree 2 , this implies that $\lambda \equiv \lambda^{\prime}$ $(\bmod I)$, as claimed.

Now consider the second case, i.e., assume that $w_{1}>0$ and $w_{2}^{\prime}>0$. Then $X_{h}$ divides $\mu$ and $Y_{k^{\prime}}$ divides $\nu^{\prime}$ for some $h, k^{\prime} \geq 1$. Set $\bar{\lambda}=X_{h-1} \lambda / X_{h}$ and $\bar{\lambda}^{\prime}=Y_{k^{\prime}-1} \lambda^{\prime} / Y_{k^{\prime}}$. Then the monomials $\bar{\lambda}$ and $\bar{\lambda}^{\prime}$ fulfill the assumption, and their weight is $w-1$. Hence induction applies to them, which allows us to conclude that they are congruent modulo $I$. Thus

$$
X_{h-1} Y_{k^{\prime}} \lambda \equiv X_{h} Y_{k^{\prime}-1} \lambda^{\prime} \quad(\bmod I) .
$$

On the other hand we have that $X_{h} Y_{k^{\prime}-1} \equiv X_{h-1} Y_{k^{\prime}}(\bmod I)$, which implies that

$$
X_{h} Y_{k^{\prime}-1} \lambda^{\prime} \equiv X_{h-1} Y_{k^{\prime}} \lambda^{\prime} \quad(\bmod I) .
$$

Hence

$$
X_{h-1} Y_{k^{\prime}} \lambda \equiv X_{h-1} Y_{k^{\prime}} \lambda^{\prime}(\bmod I),
$$

which, as above, implies that $\lambda \equiv \lambda^{\prime}(\bmod I)$, as claimed. This completes the proof.

Corollary 1.2. Let $i$ and $j$ be indices such that $1 \leq i<j \leq d$. Then $B_{n_{i}, n_{j}}$ belongs to the ideal of $S$ generated by the $(i)$-minors, the $(j)$ minors and the $(i, j)$-minors.

Proof. In view of Lemma 1.1, it suffices to note that all monomials of $B_{n_{i}, n_{j}}$ have the same weight $m_{i, j}$, the same $i$-degree $p_{i, j}$ and the same $j$-degree $q_{i, j}$, and that the sum of their coefficients is zero. 
Lemma 1.3. Let $i$ and $j$ be indices such that $1 \leq i<j \leq d$. Let $M$ be a $(i, j)$-minor. Set $m=\operatorname{lcm}\left(n_{i}, n_{j}\right)$. Then

$$
M^{m} \in\left(B_{n_{i}, n_{j}}\right)+I_{i}+I_{j} .
$$

Proof. We first introduce some notation that will simplify our argumentation. Consider the following matrix of indeterminates over $R$ :

$$
A^{\prime}=\left(\begin{array}{llll|llll}
X_{0} & X_{1} & \cdots & X_{a-1} & Y_{0} & Y_{1} & \cdots & Y_{b-1} \\
X_{1} & X_{2} & \cdots & X_{a} & Y_{1} & Y_{2} & \cdots & Y_{b}
\end{array}\right) .
$$

Let $I$ and $J$ be the ideals of $R\left[X_{0}, \ldots, X_{a}, Y_{0}, \ldots, Y_{b}\right]$ generated by the (1)-minors and the (2)-minors of $A^{\prime}$, respectively. Further, let $m=\operatorname{lcm}(a, b)$, and let $p, q$ be such that $m=p a=q b$. Then, for all $\alpha=0, \ldots, m$, let $\alpha=c p+r=e q+f$, where $0 \leq r<p$ and $0 \leq f<q$. Finally, let

$$
B_{a, b}=B_{a, b}(X, Y)=\sum_{\alpha=0}^{m}(-1)^{\alpha}\left(\begin{array}{c}
m \\
\alpha
\end{array}\right) X_{a-c}^{p-r} X_{a-c-1}^{r} Y_{e}^{q-f} Y_{e+1}^{f} .
$$

We show that, for all indices $i, u$ such that $0 \leq i \leq a-1,0 \leq u \leq b-1$,

$$
\begin{aligned}
& \left(X_{i+1} Y_{u}-X_{i} Y_{u+1}\right)^{m} \\
& \quad \equiv X_{a}^{p i} X_{0}^{m-p i-p} Y_{b}^{q u} Y_{0}^{m-q u-q} B_{a, b} \quad(\bmod I+J) .
\end{aligned}
$$

This will imply the claim. In order to prove (1.1) it suffices to show that, for all $\alpha=0, \ldots, m$,

$$
\begin{aligned}
X_{i}^{\alpha} X_{i+1}^{m-\alpha} & \equiv X_{a}^{p i} X_{0}^{m-p i-p} X_{a-c}^{p-r} X_{a-c-1}^{r} \quad(\bmod I) \\
Y_{u}^{m-\alpha} Y_{u+1}^{\alpha} & \equiv Y_{b}^{q u} Y_{0}^{m-q u-q} Y_{e}^{q-f} Y_{e+1}^{f} \quad(\bmod J) .
\end{aligned}
$$

Now the monomials in (1.2) both have degree $m$ and weight $m(i+1)-\alpha$; the monomials in (1.3) both have degree $m$ and weight $m u+\alpha$. In view of Lemma 1.1, this shows that relations (1.2) and (1.3) are true, which completes the proof.

Lemma 1.4. Let $i, j, k$ be indices such that $1 \leq i<j \leq d$ and $k$ is different from $i, j$ (say, it is greater than both). Then for every index $h$ such that $0 \leq h \leq n_{k}, X_{k, h} B_{n_{i}, n_{j}}$ belongs to the ideal generated by all $(i, k)$-minors and all $(j, k)$-minors. 
Proof. We refer to the notation introduced in the proof of Lemma 1.3. Consider the following matrix of indeterminates over $R$ :

$$
A^{\prime \prime}=\left(\begin{array}{llll|llll|llll}
X_{0} & X_{1} & \cdots & X_{a-1} & Y_{0} & Y_{1} & \cdots & Y_{b-1} & Z_{0} & Z_{1} & \cdots & Z_{g-1} \\
X_{1} & X_{2} & \cdots & X_{a} & Y_{1} & Y_{2} & \cdots & Y_{b} & Z_{1} & Z_{2} & \cdots & Z_{g}
\end{array}\right)
$$

Let $J_{X Z}$ and $J_{Y Z}$ be the ideals of $R\left[X_{0}, \ldots, X_{a}, Y_{0}, \ldots, Y_{b}, Z_{0}, \ldots, Z_{g}\right]$ generated by the $(1,3)$-minors and by the $(2,3)$-minors of $A^{\prime \prime}$, respectively. Let $h$ be an index such that $0 \leq h \leq g$. We show that $Z_{h} B_{a, b} \equiv 0\left(\bmod J_{X Z}+J_{Y Z}\right)$. Note that all monomial terms in $B_{a, b}$ are of the form $\mu \nu$, where $\mu$ is a monomial in the entries of the first block of $A^{\prime \prime}, \nu$ is a monomial in the entries of the second block of $A^{\prime \prime}$, respectively, $\mu$ has degree $p, \nu$ has degree $q$, and $w(\mu \nu)=w(\mu)+w(\nu)=m$.

On the other hand, the sum of the integer coefficients in $B_{a, b}$ is 0 . Hence it suffices to show that all monomials of the form $Z_{h} \mu \nu$, with $\mu$ and $\nu$ fulfilling the above properties, are pairwise congruent modulo $J_{X Z}+J_{Y Z}$. We show this by proving that all of these monomials are congruent to $Z_{h} X_{0}^{p} Y_{b}^{q}$ modulo $J_{X Z}+J_{Y Z}$. Let $\lambda=Z_{h} \mu \nu$ be such a monomial. First assume that $h<g$. In this case we proceed by ascending induction on $w=w(\mu)$. If $w=0$, then the constraints on weight and degree imply that $\lambda=Z_{h} X_{0}^{p} Y_{b}^{q}$, so that the claim is trivially true. Now assume that $w(\mu)>0$, and suppose that the claim is true whenever the weight of $\mu$ is smaller. Let $\mu=X_{i_{1}}^{s_{1}} X_{i_{1}-1}^{s_{2}}$ and $\nu=Y_{j_{1}}^{t_{1}} Y_{j_{1}+1}^{t_{2}}$. Then $\mu$ is not a power of $X_{0}$. Hence we may assume that $i_{1}>0$ and $s_{1}>0$. Set $\mu^{\prime}=X_{i_{1}}^{s_{1}-1} X_{i_{1}-1}^{s_{2}+1}$, which, like $\mu$, is a monomial of degree $p$. Since $Z_{h} X_{i_{1}}-Z_{h+1} X_{i_{1}-1} \in J_{X Z}$, we have that $Z_{h} \mu \equiv Z_{h+1} \mu^{\prime}\left(\bmod J_{X Z}\right)$, so that $\lambda \equiv Z_{h+1} \mu^{\prime} \nu\left(\bmod J_{X Z}\right)$. Now $w(\mu)>0$ implies that $w(\nu)<m$. It follows that $\nu$ is not a power of $Y_{b}$. Hence we may assume that $t_{1}>0$. Set $\nu^{\prime}=Y_{j_{1}}^{t_{1}-1} Y_{j_{1}+1}^{t_{2}+1}$, which is a monomial of degree $q$. Since $Z_{h+1} Y_{j_{1}}-Z_{h} Y_{j_{1}+1} \in J_{Y Z}$, we have that $Z_{h+1} \nu \equiv Z_{h} \nu^{\prime}\left(\bmod J_{Y Z}\right)$, so that $Z_{h+1} \mu^{\prime} \nu \equiv Z_{h} \mu^{\prime} \nu^{\prime}\left(\bmod J_{Y Z}\right)$. Set $\lambda^{\prime}=Z_{h} \mu^{\prime} \nu^{\prime}$. It follows that $\lambda \equiv \lambda^{\prime}$ modulo $J_{X Z}+J_{Y Z}$. Now $w\left(\mu^{\prime}\right)=w(\mu)-1$ and $w\left(\nu^{\prime}\right)=w(\nu)+1$, whence $w\left(\mu^{\prime}\right)+w\left(\nu^{\prime}\right)=$ $w(\mu)+w(\nu)=m$. Since $\mu^{\prime}$ has degree $p$ and $\nu^{\prime}$ has degree $q$, it follows that induction applies to $\lambda^{\prime}$, so that $\lambda \equiv Z_{h} X_{0}^{p} Y_{b}^{q}\left(\bmod J_{X Z}+J_{Y Z}\right)$, as desired. The case where $h=g$ can be treated similarly, by descending induction on $w(\mu)$. This completes the proof. 
For all $s=3, \ldots, 2 d-1$ let

$$
G_{s}=\sum_{i+j=s} B_{n_{i}, n_{j}}^{c_{i j}},
$$

where the positive integers $c_{i j}$ are those defined in [1, page 1651], in the following way. For all $k=3, \ldots, 2 d-1$, let $r_{k}=\operatorname{lcm}\left\{p_{i, j}+q_{i, j} \mid i+j=\right.$ $k\}$, and, whenever $i+j=k$, set $c_{i, j}=r_{k} /\left(p_{i, j}+q_{i, j}\right)$.

In order to define $B_{n_{i}, n_{j}}$ even in the case where $i$ or $j$ is greater than $d$, we imagine that the matrix $A$ is prolonged, to the right, by addition of a suitable number of blocks formed by two 0 columns. This will also allow us to consider the $(i)$-minors and the $(i, j)$ minors for the same values of $i$ and $j$.

2. Main theorem. We can now prove our main result.

Theorem 2.1. Let $L$ be the ideal of $S$ generated by all elements $F_{i, h}$ and $G_{s}$. Then $D=\sqrt{L}$.

Proof. It suffices to show that every minor of $A$ belongs to the radical of the ideal $J=\sum_{i=1}^{d} I_{i}+\left(G_{3}, \ldots, G_{2 d-1}\right)$. This is certainly true for the pure minors, since, for all $i=1, \ldots, d$, every $(i)$-minor belongs to $I_{i}$.

Now we show the claim for the non-pure minors. Let $i, j$ be indices such that $1 \leq i<j \leq d$, and set $\ell=i+j$. We show that every $(i, j)$-minor $M$ belongs to the radical of $J_{\ell}=\sum_{i=1}^{d} I_{i}+\left(G_{3}, \ldots, G_{\ell}\right)$. We proceed by double induction on $\ell$ and $i$. Note that $G_{3}=B_{n_{1}, n_{2}}$. Hence $J_{3}=\sum_{i=1}^{d} I_{i}+\left(B_{n_{1}, n_{2}}\right)$. If $\ell=3$, then $i=1$ and $j=2$, and by Lemma 1.3 it thus follows that $M \in \sqrt{J}_{3}$, which proves the induction basis.

Now suppose that $\ell>3$ and that the claim is true for all smaller values of $\ell$. First let $i=1, j=\ell-1$. From Lemma 1.3 we know that $M^{m+1} \in\left(M B_{n_{1}, n_{\ell-1}}\right)+I_{1}+I_{\ell-1}$. Hence

$$
M \in \sqrt{\left(X_{1, h} B_{n_{1}, n_{\ell-1}}, X_{1, k} B_{n_{1}, n_{\ell-1}}\right)+I_{1}+I_{\ell-1}}
$$

for some indices $h, k$. Let $u, v$ be indices such that $u<v, u+v=\ell$ and $(1, \ell-1) \neq(u, v)$. Then $1<u$ and $1<v<\ell-1$. But, according to Lemma 1.4, $X_{1, h} B_{n_{u}, n_{v}}$ and $X_{1, k} B_{n_{u}, n_{v}}$ belong to the ideal of $S$ 
generated by all $(1, u)$-minors and all $(1, v)$-minors. Since $1+u$ and $1+v$ are both less than $\ell$, by induction we have that this ideal is contained in the radical of $J_{\ell}$. It follows that

$$
\begin{gathered}
X_{1, h} B_{n_{1}, n_{\ell-1}}^{c_{1 \ell-1}}=X_{1, h} G_{\ell}-\sum_{\begin{array}{c}
u+v=\ell \\
(u, v) \neq(1, \ell-1)
\end{array}} X_{1, h} B_{n_{u}, n_{v}}^{c_{u v}} \in \sqrt{J_{\ell}}, \\
X_{1, k} B_{n_{1}, n_{\ell-1}}^{c_{1 \ell-1}}=X_{1, k} G_{\ell}-\sum_{\begin{array}{c}
u+v=\ell \\
u+v) \neq(1, \ell-1)
\end{array}} X_{1, k} B_{n_{u}, n_{v}}^{c_{u v}} \in \sqrt{J_{\ell}},
\end{gathered}
$$

and this, together with (2.1), implies that

$$
M \in \sqrt{J_{\ell}} .
$$

This shows that all $(1, \ell-1)$-minors belong to $\sqrt{J}_{\ell}$. Since, according to Corollary 1.2, $B_{n_{1}, n_{\ell-1}}$ belongs to the ideal generated by all the (1)minors, $(\ell-1)$-minors and $(1, \ell-1)$-minors, it follows that $B_{n_{1}, n_{\ell-1}} \in$ $\sqrt{J_{\ell}}$. Hence

$$
\sum_{\substack{u+v=\ell \\ 1<u<v}} B_{n_{u}, n_{v}}^{c_{u v}}=G_{\ell}-B_{n_{1}, n_{\ell-1}}^{c_{1 \ell-1}} \in \sqrt{J_{\ell}} .
$$

Now let $u$ and $v$ be indices such that $1<u<v$ and $u+v=\ell$, and suppose that, for all indices $i, j$ such that $i<j, i+j=\ell$, and $i<u$, all $(i, j)$-minors belong to $\sqrt{J_{\ell}}$. Then, by Corollary 1.2 , for all these indices $i, j$, we have that $B_{n_{i}, n_{j}} \in \sqrt{J_{\ell}}$, so that

$$
H_{u v}:=\sum_{\substack{i+j=\ell \\ i<u}} B_{n_{i}, n_{j}}^{c_{i j}} \in \sqrt{J_{\ell}} .
$$

We show that all $(u, v)$-minors belong to $\sqrt{J_{\ell}}$. Let $M$ be such a minor. Then, by Lemma 1.3 ,

$$
M \in \sqrt{\left(X_{u, h} B_{n_{u}, n_{v}}, X_{u, k} B_{n_{u}, n_{v}}\right)+I_{u}+I_{v}},
$$

for some indices $h$ and $k$. On the other hand,

$$
X_{u, h} B_{n_{u}, n_{v}}^{c_{u v}}=X_{u, h} G_{\ell}-X_{u, h} H_{u v}-\sum_{\substack{i+j=\ell \\ u<i<j}} X_{u, h} B_{n_{i}, n_{j}}^{c_{i j}},
$$


and

$$
X_{u, k} B_{n_{u}, n_{v}}^{c_{u v}}=X_{u, k} G_{\ell}-X_{u, k} H_{u v}-\sum_{\substack{i+j=\ell \\ u<i<j}} X_{u, k} B_{n_{i}, n_{j}}^{c_{i j}}
$$

Now let $i, j$ be such that $i+j=\ell$ and $u<i<j$. Then, by Lemma 1.4, $X_{u, h} B_{n_{i}, n_{j}}$ belongs to the ideal generated by all $(u, i)$-minors and all $(u, j)$-minors. Moreover, $u+i<u+j=u+\ell-i<u+\ell-u=\ell$ and $u+j<i+j=\ell$. By induction on $\ell$ it follows that all $(u, i)$-minors and all $(u, j)$-minors belong to $\sqrt{J_{\ell}}$, so that $X_{u, h} B_{n_{i}, n_{j}}, X_{u, k} B_{n_{i}, n_{j}} \in \sqrt{J_{\ell}}$. In view of (2.2), (2.3), (2.4) and (2.5), this implies that $M \in \sqrt{J_{\ell}}$ and completes the induction step.

\section{REFERENCES}

1. L. Bădescu and G. Valla, Grothendieck-Lefschetz theory, set-theoretic complete intersections and rational normal scrolls, J. Algebra 324 (2010), 1636-1655.

2. L. Robbiano and G. Valla, On set-theoretic complete intersections in the projective space, Rend. Sem. Mat. Fisico Milano 53 (1983), 333-346.

Università degli Studi di Bari "Aldo Moro," Dipartimento di Matematica, Via Orabona 4, BARI, 70125 ItALY

Email address: margherita.barile@uniba.it 